\title{
Special issue on advances in applied artificial intelligence
}

\author{
Tibor Bosse • Mark Hoogendoorn
}

Published online: 12 October 2014

(C) Springer Science+Business Media New York 2014

Artificial Intelligence (AI) emerged in the mid-twentieth century as an academic field of study. Traditionally, much emphasis was placed on the ultimate goal of AI to create intelligent machines that are able to perform any intellectual task that a human being can. However, as the field became more mature, the AI community started distinguishing a multitude of smaller, slightly less ambitious challenges, besides only focusing on 'artificial general intelligence'. More specifically, computational techniques that 'draw their inspiration from' human intelligence (rather than replicating it), have proven to be successful in solving all kinds of practical problems. Some examples of these techniques are machine learning, agent technology, and knowledge representation. Examples of application domains include, among others, medicine, economics, and incident management. This sub-area of AI, which has its main emphasis on applications of AI techniques to solve real-life problems, is currently known under the term Applied Intelligence.

The current special issue presents an overview of the state-of-the-art in Applied Intelligence. Its main aim is to provide readers with a general interest and more insight into the wide variety of AI techniques and their application domains that exist nowadays. Although the overview is not assumed to be complete, it covers a range of topics, including cognitive modelling, machine learning, and robotics. Additionally, the issue provides researchers with a more

T. Bosse $\cdot$ M. Hoogendoorn $(\bowtie)$

Department of Computer Science, VU University Amsterdam, De Boelelaan 1081, 1081 HV Amsterdam, The Netherlands

e-mail: m.hoogendoorn@vu.nl

T. Bosse

e-mail: t.bosse@vu.nl specific interest detailed technical information about the latest developments regarding these techniques. It contains 9 papers, each of which is self-contained. Hence, readers can select any individual chapter based on their personal interests, without the need of reading other chapters.

In this special issue, several papers are included that address the modeling or learning of human behavior. These models are used to facilitate the study of such behavior, or to provide intelligent systems that interact with or support humans in a more effective way. The paper by Radianti et al., for example, describes how Bayesian Networks have been used to model the dynamics of crowds in case of disasters. In the same domain, Goodwin, Granmo, and Radianti explain how Ant Colony Optimization can be used to find suitable escape routes. Koopmanschap, Hoogendoorn and Roessingh deploy a cognitive model for situation awareness which can be incorporated in automated agents for fighter pilot training and tailor the parameters of the model using machine learning techniques.

The field of robotics is prominent within this issue as well. Cacace et al. specify a high level control scheme for execution of complex tasks for Aerial Service Vehicles. Similarly, Kim, Nakadai and Okuno provide a novel approach for robots to identify the source of a sound using two microphones. Finally, the paper authored by Gouko, Kobayashi, and Kim proposes an approach which uses reinforcement learning to establish so-called discernment behavior for a robot.

Next, Rotella, Leuzzi, and Ferilli address the domain of information retrieval and the semantic web by expanding an approach which is able to learn concept networks, such as taxonomies, from plain text and therewith allowing for a more easy access of document collections. 
The last category of papers concern more fundamental developments within Applied Intelligence. Mohammad and Nishida focus on the problem of discovering approximately recurring motif in time series, which can for instance be used for gesture recognition. Additionally, Cruz, Santos, and Cruz describe a machine learning approach to contribute to the characterization of soil stiffness.

To conclude, we would like to thank all people involved in the Journal on Applied Intelligence, and in particular the editor-in-chief, Professor Moonis Ali, for providing us the opportunity to publish this special issue. Moreover, we express our gratitude to the editorial assistant, Edita Alfred, for her efforts in preparing and publishing this volume. We also greatly thank all reviewers for their hard work in assuring the high quality of this issue. And last, but not least, we cordially thank all the authors for their interesting contributions. Without their efforts, this issue could not have been published.

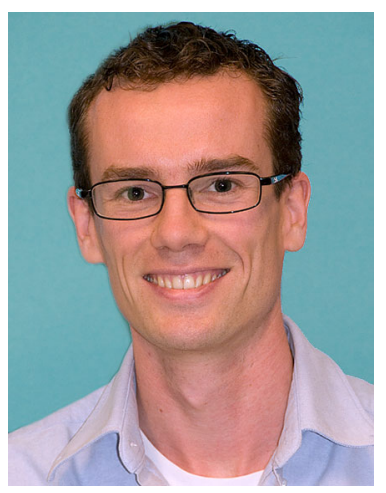

Dr. Tibor Bosse (male) is an Assistant Professor in the Agent Systems Research Group at the Department of Computer Science at VU University Amsterdam since 2006. His research focuses on computational (agent-based) modelling and simulation of human-related (social, cognitive, and physiological) processes. The developed models are used both for theoretical purposes (to gain a deeper understanding of the processes themselves) and for practical applications, for instance, in the domains of Ambient Intelligence, Education and Training, Serious Gaming, Human-Computer Interaction, and Human Factors Design. He has acquired research funding from national as well as international organisations on a regular basis. Since a number of years, his main area of interest has become Serious Gaming. Among others, he coordinates the STRESS project on simulation-based training of public service workers. Specifically, he has an interest in the application of affective computing and cognitive modelling to develop more effective games. He uses the agent paradigm in two ways, namely for the development of 1) more human-like virtual characters and 2) intelligent virtual tutors. His extensive publication list (see http://www.few.vu.nl/ tbosse/ papers.html) covers more than 150 papers in the major journals and conferences within Artificial Intelligence, Modelling and Simulation, Cognitive Science, and AI Applications, as well as a number of edited books in those areas. Also, he has served in the Organising and Programme Committees of a large number of international conferences and workshops in those areas. In particular, he is the coinitiator and main organiser of the workshop series on Human Aspects in Ambient Intelligence (HAI, which have been held annually since 2007), and he was appointed as organising chair for the 26th International Conference on Industrial, Engineering and Other Applications of Applied Intelligent Systems (IEA/AIE'13).

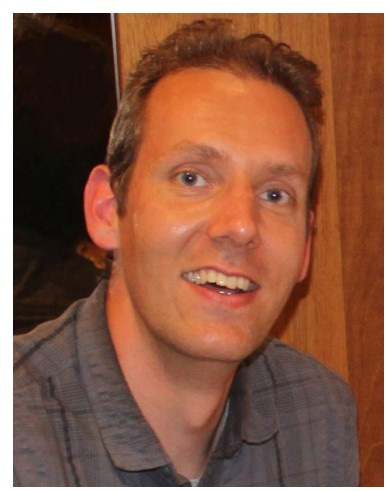

Mark Hoogendoorn is an assistant professor within the Computation Intelligence group of the Department of Computer Science at the VU University Amsterdam. Within his research, he focuses on collective adaptive systems, both from a fundamental as well as a more applied perspective. For the latter, the main domains of application include health, wellbeing, and ambient intelligence. He has an extensive publication record, and has published in both renowned international conferences as well as journals. He has acquired funding for both national and international research projects, and has been the joint coordinator of the EU FP7 funded ICT4Depression project. Furthermore, he served on numerous program committees of prominent conferences in the domain of Artificial Intelligence and has been one of the program chairs of the IEA/AIE 2013 conference. Before starting as an assistant professor, he was a visiting researcher at the University of Minnesota. He obtained his PhD degree from the VU University Amsterdam in 2007 focusing on multi-agent organizations. 